\title{
The Multiple System SZ Cam
}

\section{Gabriela Michalska ${ }^{1}$, Jiří Kubát ${ }^{2}$, Daniela Korčáková ${ }^{2}$, Adéla Kawka ${ }^{2}$, Michal Ceniga ${ }^{2,3}$, Blanka Kučerová ${ }^{3}$, and Viktor Votruba ${ }^{2,3}$}

\author{
${ }^{1}$ Instytut Astronomiczny Uniwersytetu Wrocławskiego, ul. Kopernika 11, Wrocław, Poland \\ ${ }^{2}$ Astronomický ústav AV CR, Fričova 298, 25165 Ondřejov, Czech Republic \\ ${ }^{3}$ Ústav teoretické fyziky a astrofyziky PřF MU, Kotlářská 2, 61137 Brno, Czech Republic
}

\begin{abstract}
The multiple stellar system SZ Cam is solved using the method of spectral disentrangling. $\mathrm{H} \alpha$ line profiles of three components are obtained and spectroscopic orbit elements are redetermined.
\end{abstract}

Keywords. Binaries: spectroscopic - stars: individual (SZ Cam)

\section{Introduction}

The multiple hierarchical system SZ Cam is one of the brightest members of the open cluster NGC 1502 and is the B component of the visual double ADS 2984. It is composed of four stars: an SB2 eclipsing binary, which is physically bound to an SB1 binary pair. The multiple nature of SZ Cam has been studied by many authors (Mayer et al. 1994; Lorenz et al. 1998; Harries et al. 1998; Gorda 2002). We present the results obtained from the spectroscopic analysis of the system.

\section{Data}

The spectroscopic data we used in the analysis were taken at two different sites:

- 17 new spectra obtained with the 2-m telescope at the Ondřejov Observatory between 2004 and 2006 at the coudé focus (for a description of the spectrograph see Šlechta \& Škoda 2002);

- 19 spectra provided by P. Mayer, obtained at Calar Alto Observatory in 1993 and 1995 (Lorenz et al. 1998).

\section{Analysis}

We use the KOREL code (Hadrava 1995, 1997, 2004, 2006) in our analysis. This program is a powerful tool for the decomposition of spectra, as well as for the determination of orbital parameters. For the disentangling the spectra we have chosen the H $\alpha(6564 \AA)$ line. The decomposed spectra of three components of SZ Cam, obtained for the $\mathrm{H} \alpha$ line, are shown in the Figure 1. Radial velocities for the primary and secondary components are shown in Figure 2, while for the tertiary, in Figure 3. In Table 1, the orbital parameters of the system obtained using KOREL are given.

\section{Acknowledgements}

We thank P. Mayer for providing the spectra of SZ Cam. We are grateful to P. Hadrava 


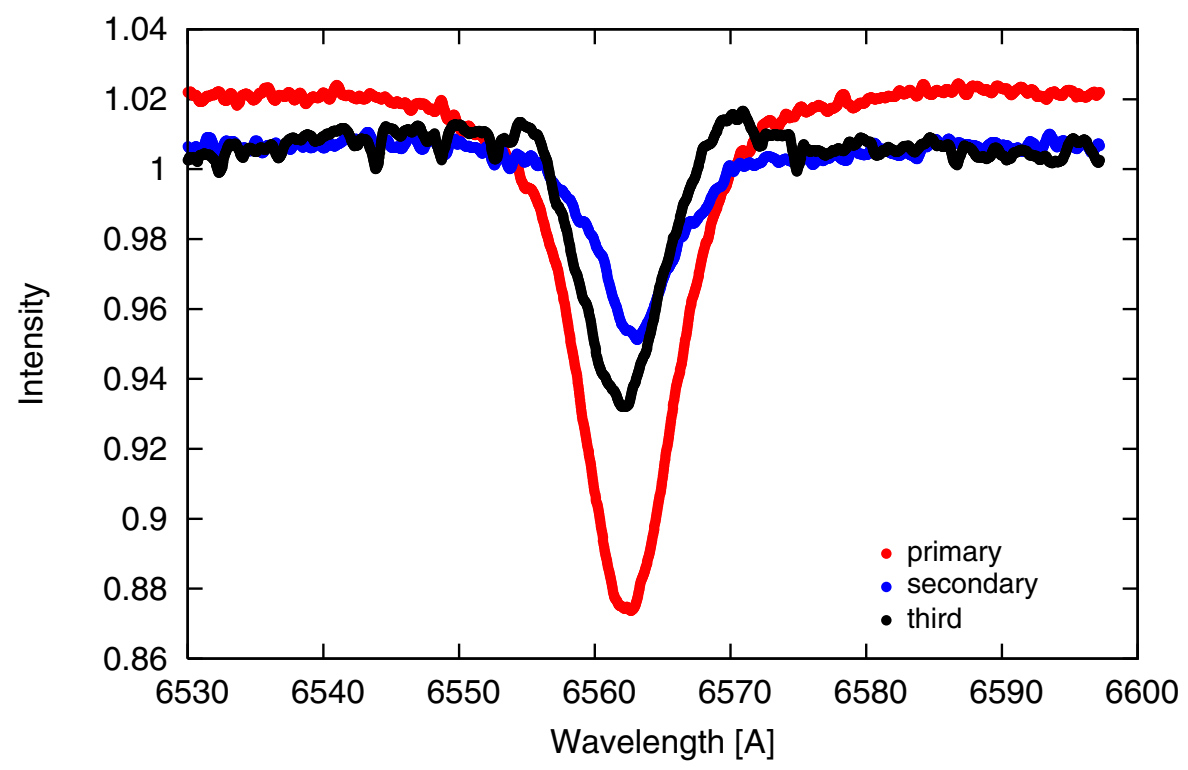

Figure 1. Decomposed H $\alpha$ spectrum of SZ Cam for the primary (red), secondary (blue), and tertiary (black) components.

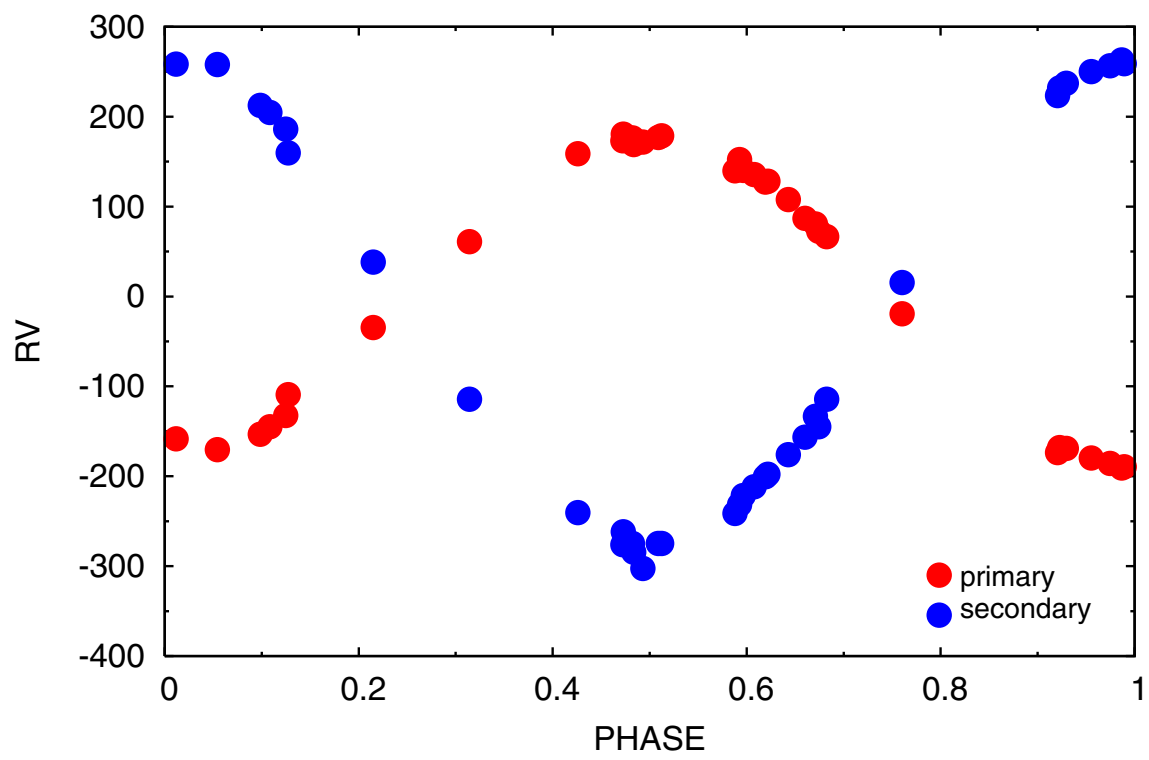

Figure 2. Radial velocities of the primary and secondary components of SZ Cam obtained with the KOREL code after disentangling the spectra.

for his help in running the KOREL code. This research was supported by the MNiI grant No. 1 P03D 01627 and by the GA grant ČR 205/04/1267. 


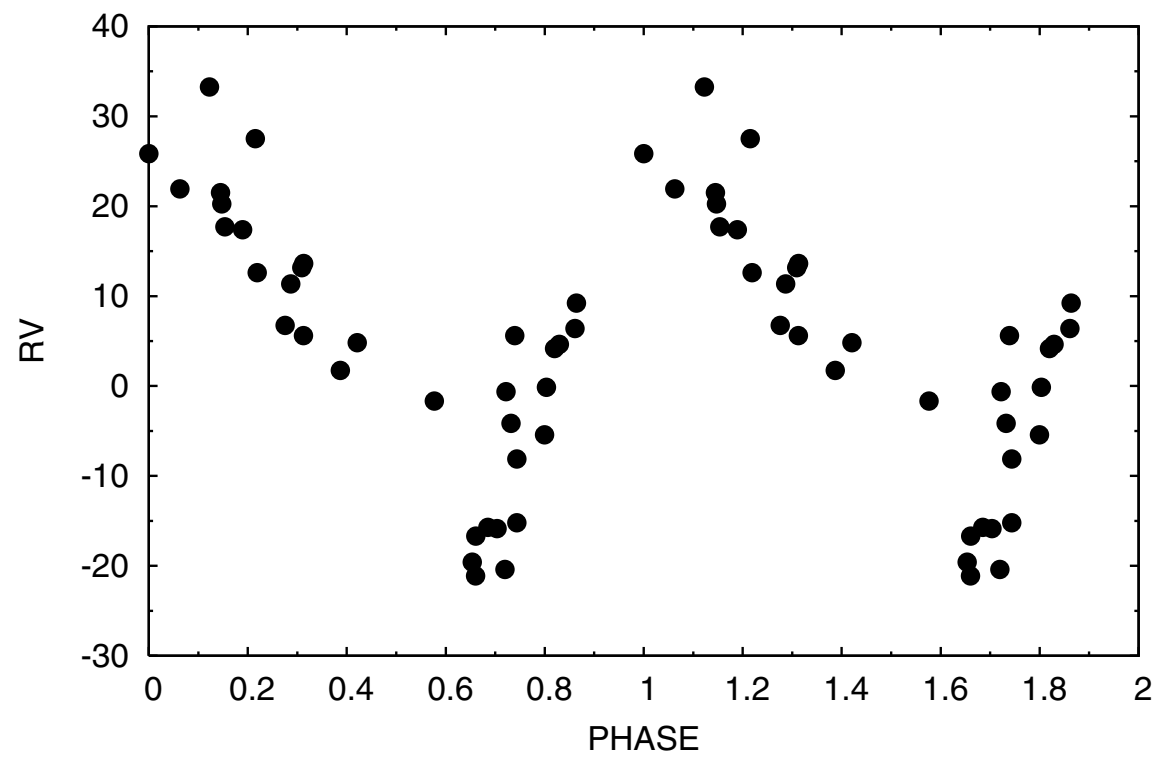

Figure 3. Radial velocity of the SB1 tertiary component of SZ Cam.

Table 1. Orbital parameters of SZ Cam.

\begin{tabular}{lc}
\hline eclipsing pair & \\
\hline$P[\mathrm{~d}]$ & 2.698401 \\
$T_{0}$ & 48933.02658 \\
$K_{1}[\mathrm{~km} / \mathrm{s}]$ & 181.591 \\
$K_{2}[\mathrm{~km} / \mathrm{s}]$ & 268.210 \\
$M_{2} / M_{1}$ & 0.68 \\
\hline $\mathrm{SB} 1$ pair & \\
\hline$P_{3}[\mathrm{~d}]$ & 2.798336 \\
$T_{\min }$ & 51478.669 \\
$K_{3}[\mathrm{~km} / \mathrm{s}]$ & 19.4 \\
$f(M)\left[\mathrm{M}_{\odot}\right]$ & 0.0021 \\
\hline
\end{tabular}

\section{References}

Gorda, S.Y. 2002, IBVS 5345

Hadrava P. 1995, A $6 A S$ 114, 393

Hadrava P. 1997, AESAS 122, 581

Hadrava P. 2004, Publ. Astron. Inst. ASCR 92, 15 (http://www.asu.cas.cz/ ^had/korel.html) Hadrava P. 2006, AESA 448, 114

Harries, T.J., Hilditch, R.W., \& Hill, G. 1998, MNRAS 295, 386

Lorenz, R., Mayer, P., \& Drechsel, H. 1998, A\&3A 332, 909

Mayer, P., Lorenz, R., Chochol, D., \& Irsmambetova T.R. 1994, A\&4A 288, L13

Šlechta, M., \& Škoda, P. 2002, Publ. Astron. Inst. ASCR 90, 9 